\title{
Role of PiCCO monitoring for the integrated management of neurogenic pulmonary edema following traumatic brain injury: A case report and literature review
}

\author{
XIAOPING LIN ${ }^{1}$, ZHIJUN XU $^{2}$, PENGFEI WANG ${ }^{2}$, YAN XU $^{3}$ and GENSHENG ZHANG ${ }^{2}$ \\ ${ }^{1}$ Department of Cardiology, ${ }^{2}$ General Intensive Care Unit, and ${ }^{3}$ Department of Critical Care Medicine, \\ Second Affiliated Hospital, Zhejiang University School of Medicine, Hangzhou, Zhejiang 310009, P.R. China
}

Received May 5, 2015; Accepted July 22, 2016

DOI: $10.3892 / e t m .2016 .3615$

\begin{abstract}
Neurogenic pulmonary edema (NPE) is occasionally observed in patients with traumatic brain injury (TBI); however, this condition is often underappreciated. NPE is frequently misdiagnosed due to its atypical clinical performance, thus delaying appropriate treatment. A comprehensive management protocol of NPE in patients with TBI has yet to be established. The current study reported the case of a 67-year-old man with severe TBI who was transferred to our intensive care unit (ICU). On day 7 after hospitalization, the patient suddenly suffered tachypnea, tachycardia, systemic hypertension and hypoxemia during lumbar cistern drainage. Intravenous diuretics, tranquilizer and glucocorticoid were administered due to suspected left heart failure attack. Chest radiography examination supported the diagnosis of pulmonary edema; however, hypotension and hypovolemia were subsequently observed. Pulse index continuous cardiac output $(\mathrm{PiCCO})$ hemodynamic monitoring and bedside echocardiography were performed, which excluded the diagnosis of cardiac pulmonary edema, and thus the diagnosis of NPE was confirmed. Goal-directed therapy by dynamic PiCCO monitoring was then implemented. In addition, levosimendan, an inotropic agent, was introduced to improve cardiac output. The patient had complete recovered from pulmonary edema and regained consciousness on day 11 of hospitalization. The current case demonstrated that PiCCO monitoring may serve a central role in the integrated management of NPE in patients with TBI. Levosimendan may be a potential medicine in treating cardiac dysfunction, along with its benefit from improving neurological function in NPE patients.
\end{abstract}

Correspondence to: Dr Gensheng Zhang, General Intensive Care Unit, Second Affiliated Hospital, Zhejiang University School of Medicine, 88 Jiefang Road, Hangzhou, Zhejiang 310009, P.R. China E-mail: zhanggensheng0122@163.com

Key words: neurogenic pulmonary edema, traumatic brain injury, pulse indicate contour cardiac output, levosimendan

\section{Introduction}

Neurogenic pulmonary edema (NPE) is characterized by abruptly increased pulmonary interstitial and alveolar fluid following central nervous system (CNS) events, including traumatic brain injury (TBI), subarachnoid hemorrhage (SAH) or spinal cord injury (1). It is a relatively rare clinical syndrome with high mortality, and is frequently misdiagnosed due to its unspecific clinical presentation. TBI contributes to the majority of injury-associated mortality and permanent disability cases worldwide (2), with the incidence of such cases reported to be $\sim 20 \%$ in patients with TBI (3). However, the incidence of NPE has reportedly increased to $50 \%$ (4) in patients succumbing within $96 \mathrm{~h}$ after suffering TBI, rendering NPE a life threatening complication in these patients.

The clinical manifestations of NPE are almost identical to those of acute respiratory distress syndrome and cardiogenic pulmonary edema, posing a great challenge for timely differential diagnosis and correct treatment decision in TBI patients (1). Although the underlying pathogenesis of NPE is poorly understood, it has been suggested that, on the basis of increased intracranial pressure, factors including direct cardiac injury, systemic sympathetic discharge, pulmonary vascular permeability and pulmonary venule adrenergic hypersensitivity may serve an essential role in NPE development $(1,5)$. These postulated mechanisms may alone or synergically drive the development of NPE, suggesting that the actual pathophysiological alteration may be complicated and that appropriate monitoring is required. Since neurohemodynamic interaction serves a central role in the development of NPE, theoretically, hemodynamic monitoring would contribute to the evaluation of the patient's condition (5). Detection of global end diastolic volume index (GEDVI), intrathoracic blood volume index (ITBVI) and extravascular lung water (EVLWI) through an advanced transpulmonary thermodilution device is known as the pulse index continuous cardiac output (PiCCO) (6). This method allows the differentiation between permeability and hydrostatic pulmonary edema, and the guidance of fluid management (6). PiCCO has also shown favorable agreement with pulmonary artery catheterization (PAC) in monitoring cardiac output (7), making it a promising strategy for handling the cardiac involvement in NPE. However, to the best of our 
knowledge, only one previous study has utilized this novel method for guiding the management of NPE (6).

Specific treatments, such as $\alpha$-adrenergic blocking agents for underlying neurological insult, have been occasionally administered in patients with NPE (8). However, an $\alpha$-adrenergic blocking agent can not be used in the case of systemic hypotension, thus an alternative treatment is required. Levosimendan, a novel positive inotropic agent, has been recommended for the treatment of acute heart failure in the European Society of Cardiology guidelines (9). In addition, a previous study unveiled the vasorelaxation ability of levosimendan on pulmonary vessels (10), suggesting that it can be used to alleviate pulmonary edema by directly reducing the hydrostatic pressure. However, its role in the management of NPE has not been investigated.

In the current study, a TBI case with NPE diagnosed when performing lumbar cistern drainage was reported. PiCCO monitoring was applied, and showed a favorable ability to interpret the pathophysiological progression and provide compelling assistance for the diagnosis and treatment of this complication. To the best of our knowledge, the present study was the first to report the use of levosimendan to improve cardiac output, and demonstrated a potential neuroprotective effect in the treatment of NPE.

\section{Case report}

The present case was approved by the Ethics Committee of the Second Affiliated Hospital, Zhejiang University School of Medicine (Hangzhou, China). Written informed consent was obtained from the patient for the publication of this manuscript.

A 67-year-old male patient was transferred to the General Intensive Care Unit (ICU) of the Second Affiliated Hospital, Zhejiang University School of Medicine (Hangzhou, China) in November 2014, 4 days after falling from a height of 2 meters. The patient was diagnosed with multiple injuries with severe TBI, spleen contusion, clavicle and multiple rib fractures at a local hospital. Upon initial admission, emergency decompressive craniectomy and hematoma removal were performed initially when progressive intracranial hypertension was suspected. A second craniotomy was performed due to increased volume of drain output on the same day. Other treatments including respiration support, antibiotics and fluid administration were also administrated. The patient had no medical history other than administration of amlodipine due to hypertension for 5 years.

During the admission in our ICU, the patient was unconscious with Glasgow Coma Scale (GCS) score (11) of 3 (E1VTM1), with possible scores ranging from 3-15; GCS scores of 3-8, 9-12 and 13-15 indicate severe, moderate and mild brain injury respectively. The patient's body temperature, pulse rate, blood pressure and respiratory rate were $38^{\circ} \mathrm{C}$, 88 beat per minute (bpm), 124/67 $\mathrm{mmHg}$ and 18 count per minute (cpm), respectively. Physical examination revealed wheezing rales of bilateral lungs upon auscultation. A head computed tomography (CT) scan showed bilateral frontal lobe and right temporal lobe contusion and laceration, subdural hematoma and SAH with post decompressive craniectomy presentation (Fig. 1). In addition, a chest X-ray scan indicated board bilateral infiltrates, consolidation and pleural effusion

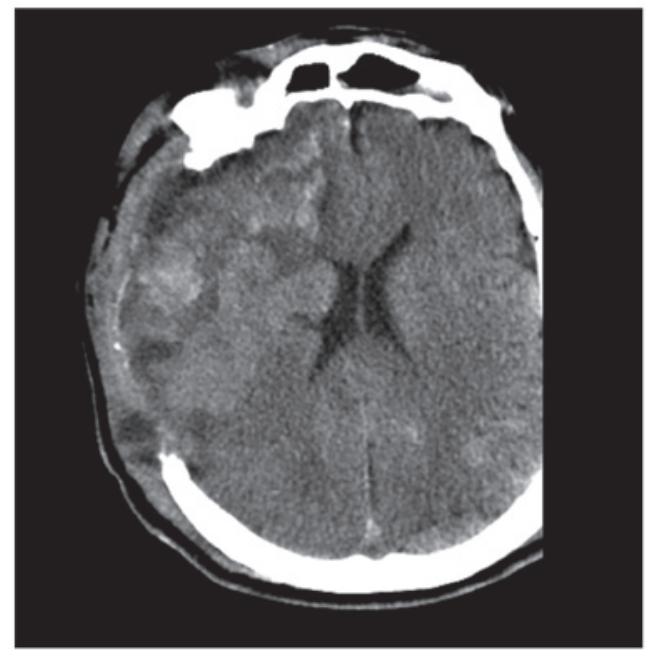

Figure 1. Head computed tomography on admission revealed bilateral frontal lobe and right temporal lobe contusion and laceration, subdural hematoma and subarachnoid hemorrhage.

(Fig. 2A). Subsequently, pulmonary infection was confirmed based on the findings of further laboratory tests, including elevated percentage of neutrophils $(91.2 \%$; normal range, $50-70 \%$ ) in complete blood counting, C-reactive protein (patient level, $92.6 \mathrm{mg} / \mathrm{l}$; normal range, $0-8 \mathrm{mg} / \mathrm{l}$ ) and serum procalcitonin (PCT; patient level, $4.2 \mathrm{ng} / \mathrm{ml}$; normal range, 0-0.05 ng/ml). Subsequently, cefoperazone/sulbactam (Pfizer, Inc., New York, NY, USA) was intravenously administered every 8 hours from days 1-3 as treatment for the pulmonary infection, and 20\% mannitol $(100 \mathrm{ml}$; Baxter Healthcare Co., Ltd., Shanghai, China) was intravenously infused every $8 \mathrm{~h}$ from days 1-11 post-admission, in order to reduce intracranial pressure. The patient remained hemodynamically stable and moderate improvement of the neurological status (GCS score, 5; E2VTM2) was observed over the next few days.

On day 7 of hospitalization, a lumbar puncture revealed substantially elevated intracranial pressure (ICP; $>300 \mathrm{mmH}_{2} \mathrm{O}$; normal range, 70-200 $\mathrm{mmH}_{2} \mathrm{O}$ ), although relevant therapies including decompressive craniectomy and osmotherapy were adequately provided. Therefore, the patient was subjected to lumbar cistern drainage. The condition of the patient was generally stable prior to the surgery, with a pulse rate, blood pressure, and respiratory rate of $94 \mathrm{bpm}$, $123 / 78 \mathrm{mmHg}$ and $22 \mathrm{cpm}$, respectively. His arterial blood peripheral oxygen saturation $\left(\mathrm{SpO}_{2}\right)$ was maintained at $100 \%$ under ventilation support of $40 \%$ fraction of inspired oxygen $\left(\mathrm{FiO}_{2}\right)$ and $5 \mathrm{cmH}_{2} \mathrm{O}$ positive end-expiratory pressure (PEEP). However, at the end of the procedure, the patient suffered sudden profuse sweating, tachypnea (25-35 cpm), tachycardia (140-90 bpm), systemic hypertension (160-200/90-120 mmHg) and decreasing $\mathrm{SpO}_{2}$ (85-95\%). Pink frothy sputum was observed upon airway aspiration, while board bilateral crackles and rales were detected upon auscultation. The diagnosis of acute left heart failure and cardiogenic pulmonary edema was initially suspected, and thus $5 \mathrm{mg}$ midazolam (Jiangsu Nhwa Pharmaceutical Co., Ltd., Xuzhou, China), $40 \mathrm{mg}$ furosemide (Kingyork, Tianjin, China), 40 mg methylprednisolone (Pfizer, Inc.) and $10 \mathrm{mg}$ morphine (Northeast Pharmaceutical Group Co., Ltd., Shenyang, China) were intravenously injected 
A

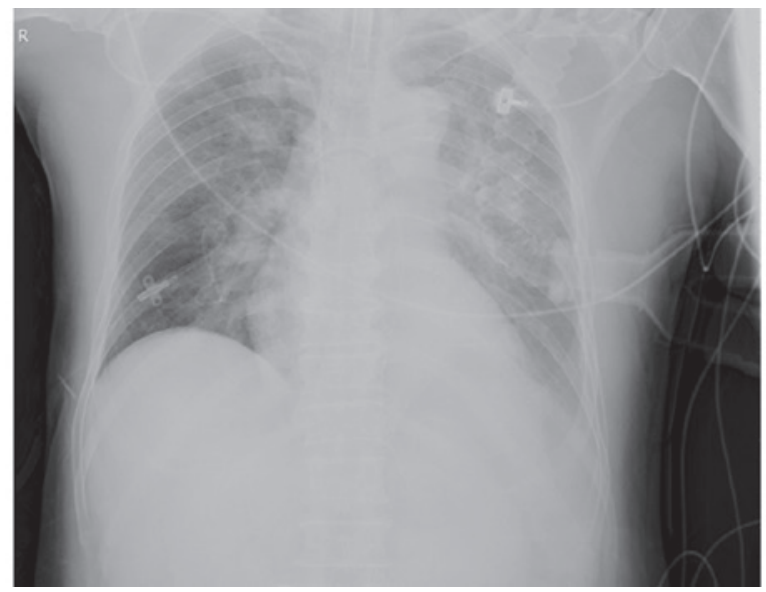

B

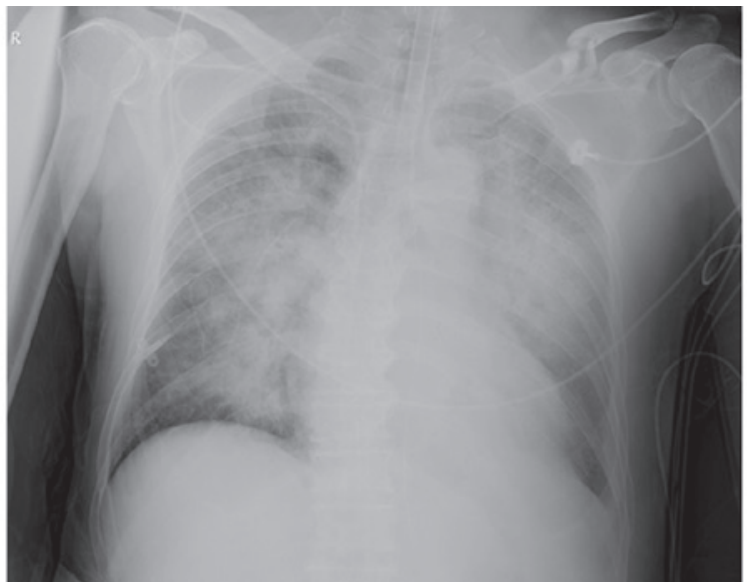

Figure 2. Chest radiography showed (A) board bilateral infiltrates, consolidation and pleural effusion on admission, and (B) typical signs of pulmonary edema with Kerley B lines and ‘bat-wing' pattern on hospitalization day 7 (neurogenic pulmonary edema onset).

following the lumbar drainage procedure, and adjustment of ventilation parameters was performed (IPPV mode; $\mathrm{FiO}_{2}$, 100\%; PEEP, $15 \mathrm{cmH}_{2} \mathrm{O}$; pressure support, $30 \mathrm{cmH}_{2} \mathrm{O}$; tidal volume, $600-700 \mathrm{ml}$; respiratory rate, $20 \mathrm{cpm})$. After $30 \mathrm{~min}$, the vital signs gradually became relatively stable, with a pulse rate of $102 \mathrm{bpm}$, blood pressure of 120/68 $\mathrm{mmHg}$, respiratory rate of $30 \mathrm{cpm}$ and $\mathrm{SpO}_{2}$ of $95-98 \%$; thus, the patient was transferred back to the general ICU.

In the ICU, the symptoms of tachypnea (32 cpm) and tachycardia (122 bpm) remained, but hypotension $(85 / 45 \mathrm{mmHg})$ was newly developed. Symptoms of hypovolemia, including cold limbs, cyanosis of the lips and oliguria, were subsequently observed, and thus norepinephrine (Grand Pharmaceutical Co. Ltd., Wuhan, China) was administered intravenously at $0.05 \mu \mathrm{g} / \mathrm{kg} / \mathrm{min}$ with an increment of $0.05 \mu \mathrm{g} / \mathrm{kg} / \mathrm{min}$ when the blood pressure was below $90 / 60 \mathrm{mmHg}$ for a total of 13 h. Arterial blood gas measurement showed a pH of 7.299, $\mathrm{CO}_{2}$ partial pressure of $46.2 \mathrm{mmHg}$ (normal range, 36-44), $\mathrm{O}_{2}$ partial pressure of $98.5 \mathrm{mmHg}$ (normal range, 75-95), blood base excess of $-3.7 \mathrm{mmol} / \mathrm{l}$ and oxygen saturation of $98.5 \%$, while receiving $100 \%$ of $\mathrm{FiO}_{2}$. Elevated levels of serum markers were also detected, including brain natriuretic peptide (BNP) of 3,438.60 pg/ml (normal range, 2-4 pg/ml), myocardial enzyme (troponin I) of $0.935 \mathrm{ng} / \mathrm{ml}$ (normal range, $<0.1 \mathrm{ng} / \mathrm{ml}$ ) and C-reactive protein (CRP) of $221.9 \mathrm{mg} / 1$. On the same day, chest X-ray imaging showed typical signs of pulmonary edema (Fig. 2B).

In order to further clarify the pathogenesis of pulmonary edema, PiCCO monitoring by transpulmonary thermodilution technique was performed (day 7). The initial PiCCO measurements revealed elevated GEDVI of $860 \mathrm{ml} / \mathrm{m}^{2}$ (normal range, $680-800 \mathrm{ml} / \mathrm{m}^{2}$ ), EVLWI of $12 \mathrm{ml} / \mathrm{kg}$ (normal range, $3-7 \mathrm{ml} / \mathrm{kg}$ ), and mild reduced cardiac index $(\mathrm{CI})$ of $2.841 / \mathrm{min} / \mathrm{m}^{2}$ (normal range, $3.0-5.01 / \mathrm{min} / \mathrm{m}^{2}$ ) combined with normal pulmonary vascular permeability index (PVPI) of 1.4 (normal range, 1-3). Thus, the diagnosis of hydrostatic-type pulmonary edema was established. Bedside two-dimensional echocardiography excluded marked left ventricular dysfunction [left ventricle ejection fraction (EF), 50\%; normal range, $50-70 \%$ ] or left abnormal ventricular wall motion, thus eliminating the possibility of cardiogenic pulmonary edema.
A diagnosis of NPE was finally established based on the aforementioned findings.

Goal-directed therapy guided with PiCCO was selected for the subsequent treatment of NPE. Fluid administration was restricted and a low dose of furosemide at $0.5 \mu \mathrm{g} / \mathrm{kg} / \mathrm{min}$ was administered intravenously for $10 \mathrm{~h}$ to achieve negative fluid balance until the EVLWI and GEDVI values reduced to the normal level. Methylprednisolone (40 mg, once daily, intravenously) was used to further relieve edema (day 7-8). A reduction in heart rate $(70-80 \mathrm{bpm})$ and respiratory rate (12-16 cpm) along with an elevation of the mean arterial pressure (83-101 mmHg) was observed, and thus a reduction of $\mathrm{FiO}_{2}$ to $40 \%$ and of norepinephrine infusion to the initial dosage $(0.05 \mu \mathrm{g} / \mathrm{kg} / \mathrm{min})$ was performed, without affecting the blood oxygen saturation. On day 8 , PiCCO demonstrated normalized EVLWI at $7 \mathrm{ml} / \mathrm{kg}$ and GEDVI at $723 \mathrm{ml} / \mathrm{m}^{2}$. However, a reduced CI $\left(2.08 \mathrm{1} / \mathrm{min} / \mathrm{m}^{2}\right)$ was still observed, whereas the stroke volume variation (SVV) was within the normal range, indicating inadequate cardiac reserve. On day 8, levosimendan, an inotropic agent, was also administered intravenously for $24 \mathrm{~h}$ to improve the cardiac output, with a maintenance dose of $0.14 \mu \mathrm{g} / \mathrm{kg} / \mathrm{min}$ (Qilu Pharmaceutical Co., Ltd., Jinan, China) subsequent to termination of norepinephrine administration, due to the patient's blood pressure stabilizing. An elevated CI of $2.79-4.06 \mathrm{l} / \mathrm{min} / \mathrm{m}^{2}$ was then determined by PiCCO monitoring during levosimendan administration, and was returned to similar levels thereafter. Repeated bedside echocardiography examinations also confirmed the improvement of left ventricular systolic function (EF, 65\%). PiCCO monitoring continued for a total of 6 days after admission, and the patient remained hemodynamically stable (Fig. 3).

On day 8 of hospitalization, a repeat chest X-ray scan revealed the complete disappearance of pulmonary edema signs (Fig. 4A). The patient regained consciousness with intermittent confusion on the day 11 of hospitalization (GCS score, 10; E3VTM6). Subsequently, the patient recovered rapidly and mechanical ventilation was ceased on the day 13 after admission. On day 16, a chest X-ray scan revealed evident improvement of lung infiltrates (Fig. 4B), along with marked reduction of serum inflammatory and cardiac biomarkers (CRP, $41.9 \mathrm{mg} / \mathrm{l}$; PCT, $1.67 \mathrm{ng} / \mathrm{ml}$; BNP, $778.8 \mathrm{pg} / \mathrm{ml}$ ), and thus 
A

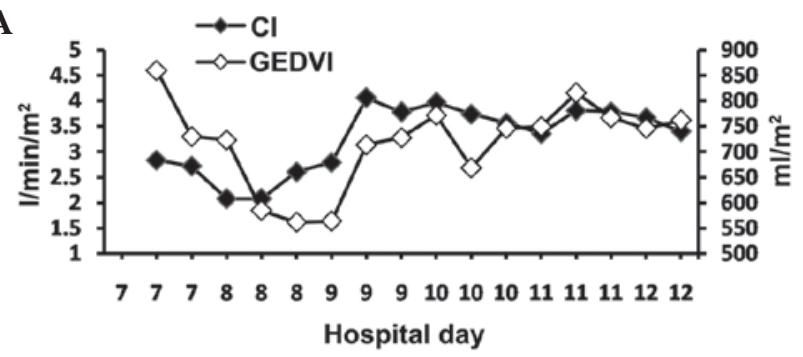

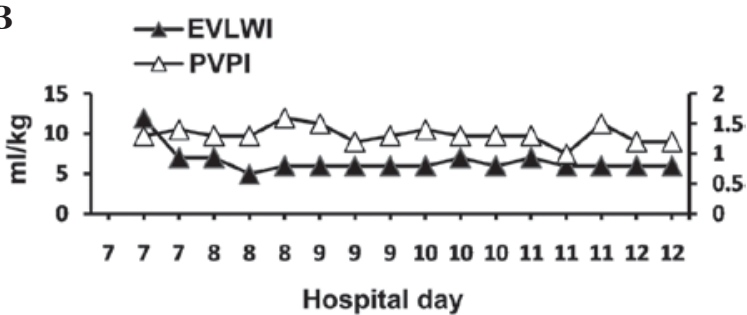

C
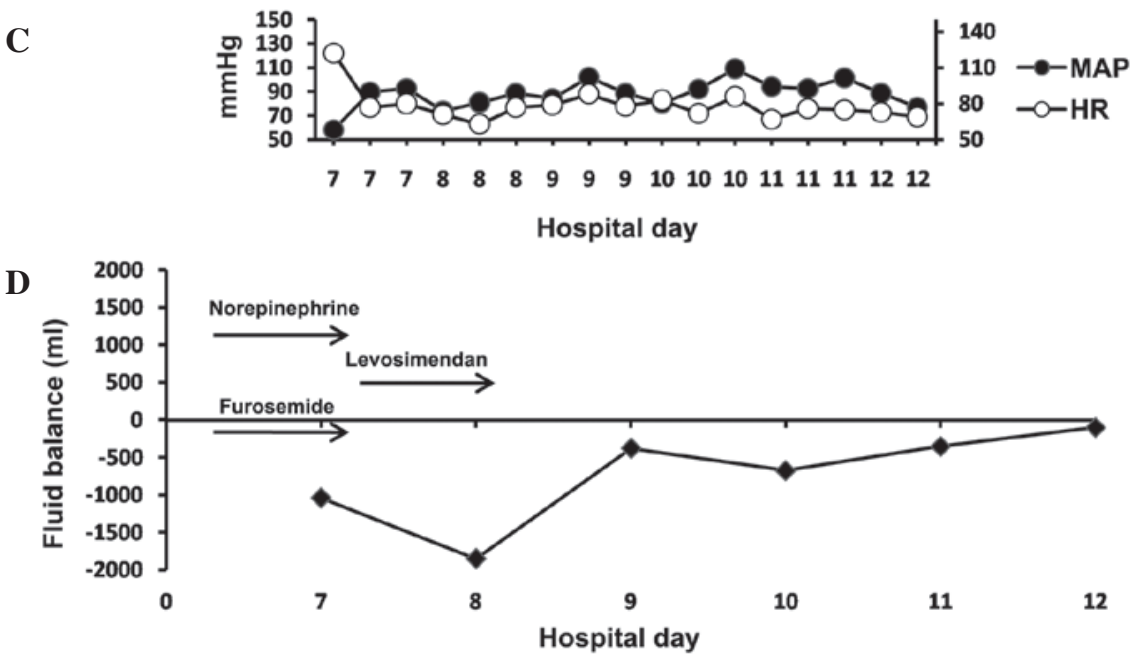

Figure 3. Changes of (A) CI and GEDVI, (B) EVLWI and PVPI, (C) MAP and HR and (D) fluid balance and major intravenous medication were reported over time during PICCO monitoring (from day 7-12 following hospital admission). PiCCO, pulse index continuous cardiac output; CI, cardiac index; GEDVI, global end diastolic volume index; EVLWI, extravascular lung water index; PVPI, pulmonary vascular permeability index; MAP, mean arterial pressure; HR, heart rate.

A

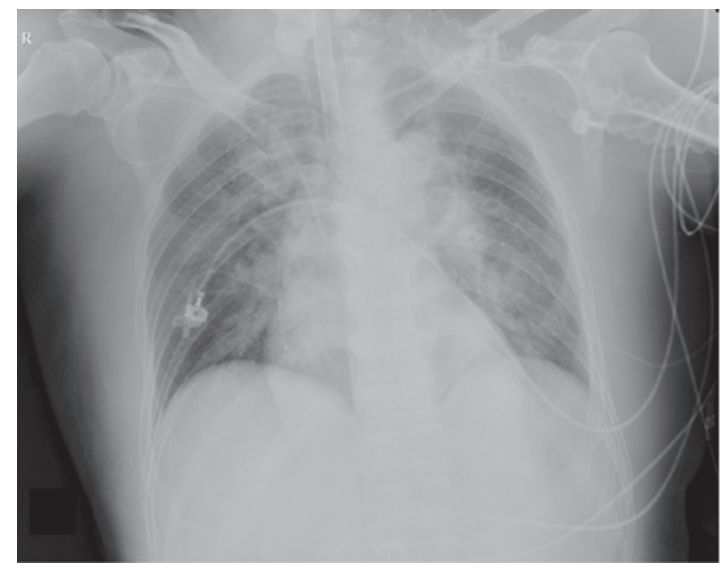

B

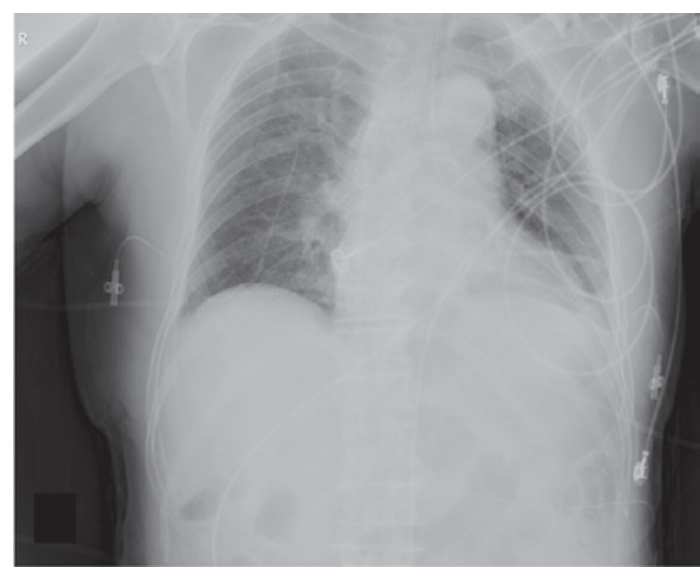

Figure 4. (A) Chest radiography on hospitalization day 8 (1 day after NPE) demonstrated no evident sign of pulmonary edema and absorbed infiltrates when compared with previous X ray images. (B) Follow-up chest radiography on hospitalization day 16 showed largely absorbed infiltrates when compared with previous chest $\mathrm{X}$ ray images. NPE, neurogenic pulmonary edema.

the antibiotic therapy was stopped. On day 22 after admission, a repeat head CT scan revealed significantly absorbed hematoma and reduced brain swelling (Fig. 5). The patient recovered uneventfully and was transferred to a rehabilitation center at 27 days after admission.

In the rehabilitation center, the patient predominantly received exercise rehabilitation. One month later, the patient regained almost complete consciousness, and ws able to walk short distances. The patient was discharged from the rehabilitation center in December 2014, requiring no additional tests or treatment. At follow-up in June 2015, the patient had recovered other than occasional confusion, and required no medication other than hypertensive drugs.

\section{Discussion}

Secondary pathophysiological changes following TBI, including alteration of cerebral blood flow, impairment of cerebrovascular autoregulation and edema formation, contribute to the elevation of ICP (12). Elevated ICP is believed to form the basis of NPE, and results in neurological compression, ischemia and disruption of the 'trigger zone', known as the 


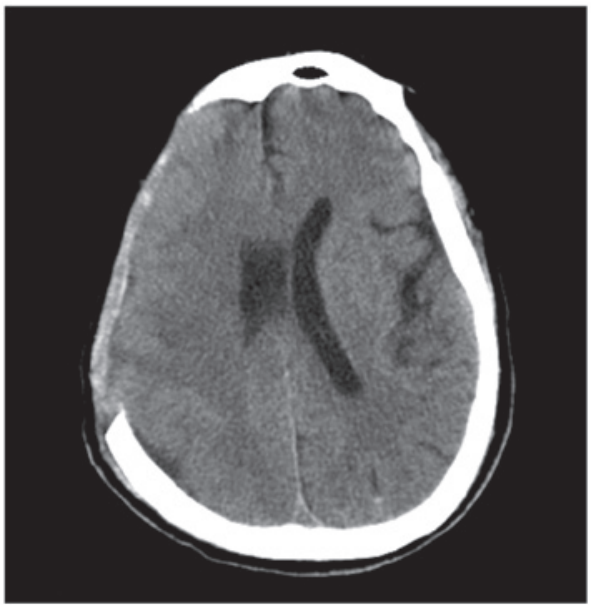

Figure 5. Follow-up head computed tomography revealed that the intracranial hematoma and subarachnoid hemorrhage were significantly absorbed and reduced brain swelling on hospitalization day 22 .

A1 and A5 groups of neurons, nuclei of the solitary tract, the area postrema, the medial reticulated nucleus and the dorsal motor vagus nucleus in the medulla oblongata $(1,5)$. There are generally two types of NPE: The 'early' form develops within minutes to hours following the injury, whereas the 'late' form develops 12-24 $\mathrm{h}$ after the injury $(1,5)$. In the case reported in the present study, the patient developed NPE during lumbar cistern drainage when significant increasing of ICP was detected. The possible explanation for this late outbreak of NPE (10 days after the initial TBI) may be that, after surviving the edema peak (usually 3-7 days post-TBI), the balance among cerebral blood flow, cerebral perfusion pressure and metabolism was restored; however, lumbar cistern drainage due to elevated ICP may cause the sudden alteration to ICP, possibly disturbing the newly established balance, inducing another brain injury and resulting in the outbreak of NPE.

Although the exact pathophysiological process remains unclear, sympathetic surge or 'catecholamine storm' is considered to be the primary mechanism of NPE $(1,5)$. Evidence from animal models revealed that elevated heart rate, and systemic and pulmonary hypertension upon CNS injury indicated a rapid activation of the sympathetic nervous system $(13,14)$. In patients with a clinical diagnosis of NPE, levels of serum catecholamine were consistently raised soon after the outbreak of the syndrome and paralleled with the alleviation of the condition $(6,8)$. The proposed pathogenesis of NPE induced by sympathetic surge includes hemodynamic disturbance, elevated pulmonary endothelial permeability, (so called 'blast theory') and direct cardiac injury $(1,5)$. These mechanisms alone or in combination, as observed in cases of NPE with Takotsubo's cardiomyopathy (15-17), give rise to the hydrostatic or permeable types of pulmonary edema, thus posing a substantial challenge for the timely recognition and management of this syndrome. In a previous case of postoperative NPE, Merenkov et al (18) reported that on-site bedside lung ultrasound was able to identify pulmonary edema, exclude cardiac involvement and guide serial fluid management. However, lung sonography testing cannot quantitatively measure the EVLWI, which is considered as the most sensitive and accurate parameter for accessing pulmonary edema (19-21). EVLWI was also shown to be closely co-associated with the ICP level in NPE (22), rendering it a reliable parameter for the precise fluid and ICP management. The newly developed PiCCO monitoring system, based on the thermodilution technique, is extensively used for the assessment of EVLWI and the estimation of intrathoracic volumes (GEDV and ITBV). PiCCO has also been proven to show favorable accordance for measuring cardiac output with the traditional 'gold standard' measurement by PAC in various groups of patients $(7,23,24)$, and to be less invasive. Mutoh et al $(6)$ demonstrated that, with combination of PVPI, GEDV and cardiac output monitoring, PiCCO was able to differentiate between the hydrostatic and permeable types of pulmonary edema in three NPE cases with aneurismal SAH. In the present study, the patient initially presented with systemic hypertension, increased heart rate and tachypnea during lumbar cistern drainage, indicating a burst of sympathetic activation. Following treatment with intravenous diuretics and morphine and adjusting ventilation parameters under consideration of acute heart failure, the patient developed hypotension and symptoms of hypovolemia, including cold limbs, cyanosis of lips and oliguria. At that time, PiCCO monitoring was decided to assist in reaching the appropriate diagnosing and in further management. The elevated GEDVI and EVLWI, normal PVPI, exclusion of major cardiac systolic dysfunction or abnormal wall motion with bedside echocardiography, the absence of excessive fluid administration and no history of heart disease suggested a non-cardiac origin of hydrostatic pulmonary edema. NPE was the most likely etiology in the current patient. However, myocardial injury represented by mild decreased CI and elevated cardiac enzyme (troponin I) and BNP levels, was likely to contribute to systemic hypotension and aggravation of hydrostatic edema. In a previous small sample study, all the NPE cases resulting from TBI were found to present cardiac dysfunction (25). The reversible myocardial dysfunction may be caused by direct catecholamine cardiotoxicity or indirect abruptly elevated afterload. Biomarkers such as BNP alone for cardiac pulmonary edema identification and prognosis prediction can be interfered by various factors, including hypovolemia (26) and abnormal BNP released from the injured CNS lesion (27). However, BNP levels appeared to be associated with the disease status evolution in the present study patient, possibly due to a certain degree of cardiac involvement.

To date, ventilation with PEEP and ICP reduction has been proven to be the most effective modality in handling NPE $(28,29)$. However, a specific treatment protocol has not been developed. Regarding the heterogeneous hemodynamic presentation of NPE due to the predominance of specific underlying pathophysiological changes $(1,5)$, an individualized treatment decision, including fluid management, the use of inotropic and vasoactive agents, is urgently required (1,28-30). These treatments constantly interfere with each other in managing TBI patients with NPE. For instance, elevated PEEP for the lung recruitment maneuver may limit venous return, further reduce cardiac stroke volume and increase the ICP (31). In addition, fluid resuscitation to help improve cardiac and brain perfusion may increase the interstitial edema and thus impair pulmonary function (32). In the current study, rapid reduction of EVLWI and normalized GEDVI along with improved clinical situation, such as relief of respiratory failure and hemodynamic instability, was obtained under guidance with the PiCCO system (Fig. 3), without causing deterioration 
of cardiac or neurological function; this implies that intense and effective hemodynamic monitoring is the key to the optimal management.

Only a limited number of studies have described the specific treatment, such as the use of an $\alpha$-adrenergic blocking agent, for underlying adrenergic surge in patients with NPE. The successful treatment with intravenous injection of phentolamine (8) was reported in a previous case of NPE caused by an intracranial hemorrhage from a ruptured arteriovenous malformation, in which increased serum catecholamine levels were documented. However, postoperative hypotension in the present patient excluded the usage of an $\alpha$-adrenergic blocking agent. An inotropic agent was thus considered for correcting the relatively low $\mathrm{CI}$, as detected by PiCCO. Levosimendan, a calcium sensitizer, is known to effectively improve cardiac function without increasing myocardial oxygen consumption (33). Possibly owing to this unique characteristic, several clinical trials or meta-analyses have implied favorable benefits, including improved cardiac function and reduction of mortality or length of hospital stay, upon levosimendan treatment when compared with dobutamine treatment in critically ill patients or those with a cardiology setting (34-37). The neuroprotective effect of levosimendan has been recently demonstrated in a TBI in vitro model (38), and in a cerebral reperfusion and spinal cord injury in vivo model $(39,40)$. The exact mechanism is unclear, and is possibly attribute to the opening of ATP-sensitive $\mathrm{K}^{+}$ channels (mitoKATP channels) and to the suppression of nitric oxide synthase expression, cell death and inflammatory response upon the application of levosimendan (40-42). Thus, treatment with levosimendan rather than dobutamine was selected in the current study, attempting to correct cardiac dysfunction without increasing oxygen consumption and preventing further brain injury. The current patient demonstrated completely reversed CI following levosimendan administration. To the best of our knowledge, this is the first report presenting the application of levosimendan in treating the cardiac dysfunction in NPE. The improved GCS score was largely attributing to lumbar cistern drainage therapy, while levosimendan treatment may partially help accelerate the recovery of brain function with its neuroprotective ability. Future clinical trials are needed to confirm the potential role of levosimendan in managing NEP patients with TBI.

In conclusion, the present study reported a case of NPE that was successfully diagnosed and treated with dynamic monitoring of PiCCO. The study demonstrated that successful identification and control of NPE was rapidly obtained through comprehensive monitoring of serial serum biomarker tests, bedside echocardiography and, most importantly, hemodynamic monitoring. Fluid management remains the key element in treating NPE. The application of the new medication, levosimendan, has extended our experience in handling the cardiac involvement under CNS insult. Further clinical trials are required to provide solid evidence on the potential of this neuroprotective inotropic agent.

\section{Acknowledgements}

The present study was supported by grants from the General Medical and Health Research Program (no. 2013KYA085), the Research Fund for the Doctoral Program of Higher Education of China (no. 20130101120035) and the Medical Science and Technology Project of Zhejiang Province (no. 201480343).

\section{References}

1. Davison DL, Terek M and Chawla LS: Neurogenic pulmonary edema. Crit Care 16: 212, 2012.

2. Langlois JA, Rutland-Brown W and Wald MM: The epidemiology and impact of traumatic brain injury: A brief overview. J Head Trauma Rehabil 21: 375-378, 2006.

3. Bratton SL and Davis RL: Acute lung injury in isolated traumatic brain injury. Neurosurgery 40: 707-712; discussion 712, 1997.

4. Rogers FB, Shackford SR, Trevisani GT, Davis JW, Mackersie RC and Hoyt DB: Neurogenic pulmonary edema in fatal and nonfatal head injuries. J Trauma 39: 860-866; discussion 866-868, 1995.

5. Sedy J,Zicha J, Kunes J, Jendelova P and Syková E: Mechanisms of neurogenic pulmonary edema development. Physiol Res 57: 499-506, 2008.

6. Mutoh T, Kazumata K, Kobayashi S, Terasaka S and Ishikawa T: Serial measurement of extravascular lung water and blood volume during the course of neurogenic pulmonary edema after subarachnoid hemorrhage: Initial experience with 3 cases. J Neurosurg Anesthesiol 24: 203-208, 2012.

7. Friesecke S, Heinrich A, Abel P and Felix SB: Comparison of pulmonary artery and aortic transpulmonary thermodilution for monitoring of cardiac output in patients with severe heart failure: Validation of a novel method. Crit Care Med 37: 119-123, 2009.

8. Davison DL, Chawla LS, Selassie L, Tevar R, Junker C and Seneff MG: Neurogenic pulmonary edema: Successful treatment with IV phentolamine. Chest 141: 793-795, 2012.

9. McMurray JJ, Adamopoulos S, Anker SD, Auricchio A, Böhm M, Dickstein K, Falk V, Filippatos G, Fonseca C, Gomez-Sanchez MA, et al: ESC guidelines for the diagnosis and treatment of acute and chronic heart failure 2012: The Task Force for the Diagnosis and Treatment of Acute and Chronic Heart Failure 2012 of the European Society of Cardiology Developed in collaboration with the Heart Failure Association (HFA) of the ESC. Eur J Heart Fail 14: 803-869, 2012.

10. Rieg AD, Rossaint R, Verjans E, Maihöfer NA, Uhlig S and Martin C: Levosimendan Relaxes Pulmonary Arteries and Veins in Precision-Cut Lung Slices-The Role of KATP -Channels, cAMP and cGMP. PLoS One 8: e66195, 2013.

11. Teasdale G and Jennett B: Assessment of coma and impaired consciousness. A practical scale. Lancet 2: 81-84, 1974.

12. Werner $\mathrm{C}$ and Engelhard K: Pathophysiology of traumatic brain injury. Br J Anaesth 99: 4-9, 2007.

13. Brashear RE and Ross JC: Hemodynamic effects of elevated cerebrospinal fluid pressure: Alterations with adrenergic blockade. J Clin Invest 49: 1324-1333, 1970.

14. Nathan MA and Reis DJ: Fulminating arterial hypertension with pulmonary edema from release of adrenomedullary catecholamines after lesions of the anterior hypothalamus in the rat. Circ Res 37: 226-235, 1975.

15. Gekka M, Yamaguchi S, Kazumata K, Kobayashi H, Motegi H, Terasaka S and Houkin K. Hemorrhagic onset of hemangioblastoma located in the dorsal medulla oblongata presenting with tako-tsubo cardiomyopathy and neurogenic pulmonary edema: A case report. Case Rep Neurol 6: 68-73, 2014.

16. Manto A, De Gennaro A, Manzo G, Serino A, Quaranta G and Cancella C: Early endovascular treatment of aneurysmal subarachnoid hemorrhage complicated by neurogenic pulmonary edema and Takotsubo-like cardiomyopathy. Neuroradiol J 27: 356-360, 2014.

17. Inamasu J, Nakatsukasa M, Mayanagi K, Miyatake S Sugimoto K, Hayashi T, Kato Y and f Hirose Y: Subarachnoid hemorrhage complicated with neurogenic pulmonary edema and takotsubo-like cardiomyopathy. Neurol Med Chir (Tokyo) 52: 49-55, 2012.

18. Merenkov VV, Kovalev AN and Gorbunov VV: Bedside lung ultrasound: A case of neurogenic pulmonary edema. Neurocrit Care 18: 391-394, 2013.

19. Patroniti N, Bellani G, Maggioni E, Manfio A, Marcora B and Pesenti A: Measurement of pulmonary edema in patients with acute respiratory distress syndrome. Crit Care Med 33: 2547-2554, 2005.

20. Berkowitz DM, Danai PA, Eaton S, Moss M and Martin GS: Accurate characterization of extravascular lung water in acute respiratory distress syndrome. Crit Care Med 36: 1803-1809, 2008. 
21. Craig TR, Duffy MJ, Shyamsundar M, McDowell C, McLaughlin B, Elborn JS and McAuley DF: Extravascular lung water indexed to predicted body weight is a novel predictor of intensive care unit mortality in patients with acute lung injury. Crit Care Med 38: 114-120, 2010.

22. Gupta YK, Chugh A, Kacker V, Mehta VS and Tandon PN: Development of neurogenic pulmonary edema at different grades of intracranial pressure in cats. Indian J Physiol Pharmacol 42 : 71-80, 1998.

23. Goedje O, Hoeke K, Lichtwarck-Aschoff M, Faltchauser A Lamm P and Reichart B: Continuous cardiac output by femoral arterial thermodilution calibrated pulse contour analysis: Comparison with pulmonary arterial thermodilution. Crit Care Med 27: 2407-2412, 1999.

24. Della Rocca G, Costa MG, Coccia C, Pompei L, DiMarco P Vilardi V and Pietropaoli P: Cardiac output monitoring: Aortic transpulmonary thermodilution and pulse contour analysis agree with standard thermodilution methods in patients undergoing lung transplantation. Can J Anaesth 50: 707-711, 2003.

25. Bahloul M, Chaari AN, Kallel H, Khabir A, Ayadi A, Charfeddine H, Hergafi L, Chaari AD, Chelly HE, Ben Hamida C, et al: Neurogenic pulmonary edema due to traumatic brain injury: Evidence of cardiac dysfunction. Am J Crit Care 15: 462-470, 2006.

26. Dorhout Mees SM, Hoff RG, Rinkel GJ, Algra A and van den Bergh WM: Brain natriuretic peptide concentrations after aneurysmal subarachnoid hemorrhage: Relationship with hypovolemia and hyponatremia. Neurocrit Care 14: 176-181, 2011.

27. Sviri GE, Soustiel JF and Zaaroor M: Alteration in brain natriuretic peptide (BNP) plasma concentration following severe traumatic brain injury. Acta Neurochir (Wien) 148: 529-533; discussion 533, 2006.

28. Fontes RB, Aguiar PH, Zanetti MV, Andrade F, Mandel M and Teixeira MJ: Acute neurogenic pulmonary edema: Case reports and literature review. J Neurosurg Anesthesiol 15: 144-150, 2003

29. Baumann A, Audibert G, McDonnell J and Mertes PM: Neurogenic pulmonary edema. Acta Anaesthesiol Scand 51: 447-455, 2007.

30. Busl KM and Bleck TP: Neurogenic Pulmonary Edema. Crit Care Med 43: 1710-1715, 2015

31. Videtta W, Villarejo F, Cohen M, Domeniconi G, Santa Cruz R, Pinillos O, Rios F and Maskin B: Effects of positive end-expiratory pressure on intracranial pressure and cerebral perfusion pressure. Acta Neurochir Suppl 81: 93-97, 2002.
32. Matsuyama T, Okuchi K, Nishiguchi T, Seki T and Murao Y: Neurogenic pulmonary edema caused by a medulla oblongata lesion after head trauma. J Trauma 63: 700-702, 2007.

33. Kersten JR, Montgomery MW, Pagel PS and Warltier DC: Levosimendan, a new positive inotropic drug, decreases myocardial infarct size via activation of K (ATP) channels. Anesth Analg 90: $5-11,2000$.

34. Huang X, Lei S, Zhu MF, Jiang RL, Huang LQ, Xia GL and Zhi YH: Levosimendan versus dobutamine in critically ill patients: A meta-analysis of randomized controlled trials. J Zhejiang Univ Sci B 14: 400-415, 2013.

35. Delaney A, Bradford C, McCaffrey J, Bagshaw SM and Lee R: Levosimendan for the treatment of acute severe heart failure: A meta-analysis of randomised controlled trials. Int J Cardiol 138 281-289, 2010.

36. Landoni G, Biondi-Zoccai G, Greco M, Greco T, Bignami E, Morelli A, Guarracino F and Zangrillo A: Effects of levosimendan on mortality and hospitalization. A meta-analysis of randomized controlled studies. Crit Care Med 40: 634-646, 2012.

37. Oner E, Erturk M, Birant A, Kurtar Mansiroglu A, Akturk IF, Karakurt H, Yalcin AA, Uzun F, Somuncu MU and Yildirim A: Assessment of sustained effects of levosimendan and dobutamine on left ventricular systolic functions by using novel tissue Doppler derived indices in patients with advanced heart failure. Cardiol J 22: 87-93, 2014.

38. Roehl AB, Hein M, Loetscher PD, Rossaint J, Weis J, Rossaint R and Coburn M: Neuroprotective properties of levosimendan in an in vitro model of traumatic brain injury. BMC Neurol 10: 97, 2010.

39. Hein M, Zoremba N, Bleilevens C, Bruells C, Rossaint R and Roehl AB: Levosimendan limits reperfusion injury in a rat middle cerebral artery occlusion (MCAO) model. BMC Neurol 13: 106, 2013.

40. Lafci B, Yasa H, Ilhan G, Ortac R, Yilik L, Kestelli M, Goktogan T and Gurbuz A: Protection of the spinal cord from ischemia: Comparative effects of levosimendan and iloprost. Eur Surg Res 41: 1-7, 2008.

41. Das B and Sarkar C: Pharmacological preconditioning by levosimendan is mediated by inducible nitric oxide synthase and mitochondrial KATP channel activation in the in vivo anesthetized rabbit heart model. Vascul Pharmacol 47: 248-256, 2007.

42. Hönisch A, Theuring N, Ebner B, Wagner C, Strasser RH and Weinbrenner C: Postconditioning with levosimendan reduces the infarct size involving the PI3K pathway and KATP-channel activation but is independent of PDE-III inhibition. Basic Res Cardiol 105: 155-167, 2010. 